\title{
Calibrating the scales of suffering
}

\author{
Do Fish Feel Pain? \\ by Victoria Braithwaite \\ Oxford University Press: 2010. 256 pp. \\ $\$ 29.95, £ 14.99$
}

Biologist Victoria Braithwaite found herself in the media spotlight in 2003 after publishing research findings suggesting that fish feel pain. After injecting the faces of trout with bee venom and vinegar, anatomical and physiological studies revealed nerve endings called nociceptors, which respond to noxious stimulation. These interventions caused the fish to show increased breathing rates and decreased feeding behaviours that persisted for more than an hour. Fish subjected to noxious stimuli also became less fearful of a novel object in their tank, which Braithwaite suggests could be because their pain made them less attentive to unfamiliar objects.

In Do Fish Feel Pain?, Braithwaite recounts these experiments and discusses their implications, integrating a range of studies on the behaviour and cognition of fish. She is careful to point out that behavioural responses and the presence of nociceptors are necessary but insufficient criteria to demonstrate that fish experience pain. She structures her argument simply: to feel pain, fish need nociceptors to detect potentially damaging stimulation; they need to show awareness of this stimulation through a behavioural reaction that goes beyond a simple reflex; and they must indicate that they are conscious by succeeding in a complex task that requires mental representations for its solution. In showing all of these steps through varied experiments, she argues, fish feel pain.

However, the issues surrounding consciousness and pain are more complex than Braithwaite's simple syllogism implies. Fully conscious humans do not always experience pain on being exposed to noxious stimuli. For example, in the heat of battle, soldiers with terrible wounds might not report pain. By contrast, one may feel pain when no stimulus is applied; for instance, a person can report pain in an amputated limb that he or she no longer possesses. Complex tasks can also be completed without conscious awareness. People often have no insight into how they solve complex reasoning problems, such as deducing how to connect a pattern of dots with a minimal number of lines. Thus, the solution of complex

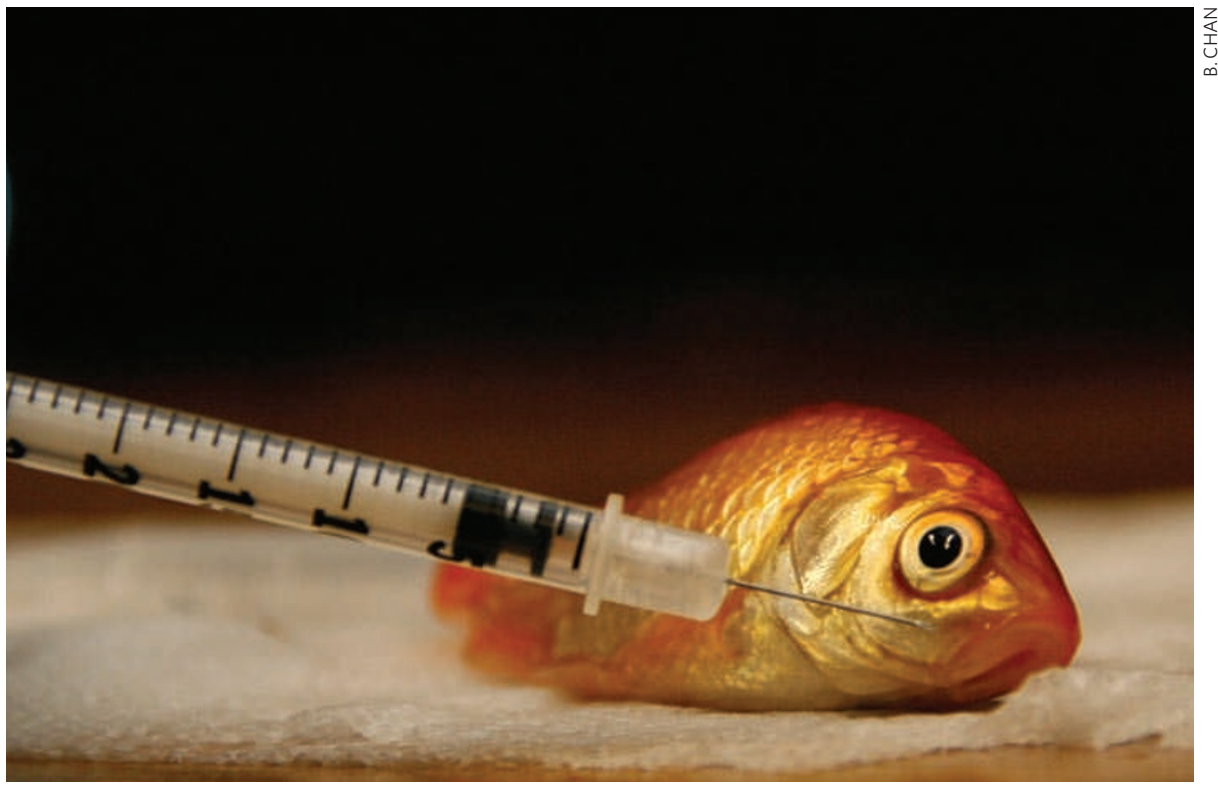

Fish have nerve endings that detect harmful stimuli, but it is difficult to prove that they feel pain.

tasks, nociception and pain experience are not strongly linked.

The task that Braithwaite appeals to as evidence that fish form conscious mental representations is called transitive inference. It is a form of reasoning in which an individual is given partial information - such as 'A is greater than $\mathrm{B}$ ' and ' $\mathrm{B}$ is greater than $\mathrm{C}$ ' - and then tested to see if they have deduced the valid inference: that A is greater than C. Ingenious experiments by then-undergraduate Logan Grosenick and his colleagues at Stanford University in Palo Alto, California, demonstrated that male African cichlids - aggressive freshwater fish - could make these judgements.

Permitted to watch contests between other fish, the cichlids observed how fish A could beat fish B in combat, and fish B could defeat fish C. To test the observer's powers of deduction, he was placed in a glass container between fishes A and C. It is known that a male cichlid who finds himself in a three-way fight will always first attack the fish he perceives to be the weakest. Thus, the observer cichlid should choose to attack fish $\mathrm{C}$ if he can solve transitive inference, as he did in these experiments.

Impressive as this demonstration is, the cichlids' behaviour does not prove that fish are conscious. There are many competing explanations for how humans and other species may solve this task, including one that relies simply on associative learning. Even if one assumes that fish solve the transitive inference problem consciously, it is a further leap to conclude from this that fish consciously experience pain.

Humans can be consciously aware of many things, from the most exquisite ecstasy to the most diabolical agonies. Yet even in a waking state, the same stimuli may pass us by without evoking any conscious awareness. It is therefore unlikely that clear answers to the question of whether animals feel pain will be obtained simply from behavioural responses.

Braithwaite is careful to weigh up the ethical implications of her conclusions. Although she thinks that fish feel pain when caught by anglers, she recognizes that the hobby of angling has positive effects, including increasing public awareness of the importance of preserving fish habitats. If angling were banned, the quality of waterways might decline, and the net outcome for fish could be negative. This recognition of the complexities of ethical decisions - and the need for nuance rather than stridency in finding the best way forward for humans and other species - redeems the book. Do Fish Feel Pain? is a fascinating excursion through the recent studies of the surprisingly complex behaviour of fish.

Clive Wynne is associate professor of psychology in the Department of Psychology, University of Florida, Gainesville, Florida 32611, USA.

e-mail:wynne@ufl.edu 